\title{
Near-IR coronagraphic imaging of the companion to HR $7672^{\star}$
}

\author{
A. Boccaletti ${ }^{1}$, G. Chauvin ${ }^{2}$, A.-M. Lagrange ${ }^{2}$, and F. Marchis ${ }^{3}$ \\ 1 LESIA, Observatoire de Paris-Meudon, 5 Pl. J. Janssen, 92195 Meudon, France \\ 2 Laboratoire d'Astrophysique de l'Observatoire de Grenoble, BP 53, 38041 Grenoble Cedex 9, France \\ e-mail: gael.chauvin@obs.ujf-grenoble.fr; anne-marie.lagrange@obs.ujf-grenoble.fr \\ ${ }^{3}$ University of California at Berkeley, Department of Astronomy, 601 Campbell Hall, Berkeley, CA 94720, USA \\ e-mail: fmarchis@astron.berkeley.edu
}

Received 1 April 2003 / Accepted 30 July 2003

\begin{abstract}
This article presents coronagraphic images of the low-mass companion to the star HR 7672 observed at the Palomar 200 inch telescope and first detected at Gemini and Keck in the $K$ band by Liu et al. (2002). We obtained additional photometry in $J(1.2 \mu \mathrm{m}), H(1.6 \mu \mathrm{m})$ and $K_{\mathrm{s}}(2.2 \mu \mathrm{m})$ bands to cover the full near-IR domain and hence to further constrain the nature of the companion. A mass estimate of $58-71 M_{J}$ is derived from evolutionary models of very low-mass objects.
\end{abstract}

Key words. stars: individual: HR7672 - stars: low-mass, brown dwarfs - instrumentation: adaptive optics techniques: high angular resolution - techniques: image processing

\section{Introduction}

Since the discovery of the first bona fide substellar objects: GD165 B (Becklin \& Zuckerman 1988) and Gl229B (Nakajima et al. 1995), an ever growing number of low-mass objects are routinely detected. Large IR surveys like 2MASS (Skrutskie et al. 19997), DENIS (Epchtein 1997) and SLOAN (York et al. 2000) or dedicated surveys in star-forming regions were initiated to explore the bottom of the main-sequence and have provided a consistent sample of more than 200 isolated low-mass stars. In the Trapezium young cluster, the presence of planetary mass candidates has been announced independently by Lucas \& Roche (2000) and Zapatero-Osorio et al. (2000) but remains doubtful since the distances of these candidates are uncertain (Tinney et al. 2003).

Two distinct classes of stars later than $\mathrm{M}$ have been identified: the so-called $\mathrm{L}$ types and $\mathrm{T}$ types. The $\mathrm{L}$ class, proposed by Kirkpatrick et al. (1999), gathers objects with effective temperatures lower than $\mathrm{M}$ dwarfs $\left(T_{\text {eff }}<2000 \mathrm{~K}\right)$ and featuring strong lines of neutral alkali elements, absence of $\mathrm{TiO}$ and $\mathrm{VO}$ absorption and stronger $\mathrm{H}_{2} \mathrm{O}$ absorption in the visible. As a consequence, the near-IR colors are becoming very red with $0.5<H-K_{\mathrm{s}}<1$ (Kirkpatrick et al. 2000). According to their age, some L dwarfs can also be Brown Dwarfs (BDs) since their mass is often below the stellar boundary $\left(0.075 M_{\odot}\right)$. Independently of the definition of the L class

Send offprint requests to: A. Boccaletti,

e-mail: Anthony.Boccaletti@obspm.fr

* Based on data collected at the Palomar 200-inch. given by Kirkpatrick et al. (1999), Martin et al. (1999) have proposed a different classification using a temperature scale derived by Basri et al. (2000). The two approaches yield different classifications for later types (beyond L2) and this discrepancy demonstrates the difficulty to tackle this new subject in astrophysics. Kirkpatrick et al. (1999) also proposed to designate Gl229 B-like objects as T dwarfs. These objects, also called "methane dwarfs", are cooler than the $\mathrm{L}$ type $\left(T_{\mathrm{eff}}<1300 \mathrm{~K}\right)$ and exhibit very strong $\mathrm{H}_{2} \mathrm{O}$ and $\mathrm{CH}_{4}$ absorption in the $H$ and $K$ bands. As a result, their color index $J-K$ and $H-K$ are very close to 0 . It is now well admitted that these 2 classes of stars correspond to the missing part at the bottom of the main sequence between $\mathrm{M}$ stars and giant planets. The study of lowmass objects either isolated or orbiting stars is of course very exciting and is definitely mandatory to further understand both the brown dwarf and planetary formation process.

Low-mass stars as well as brown dwarfs have also been studied from a theoretical point of view. However, the processes occurring in these objects appear quite complex (especially regarding the presence of dust below $T_{\mathrm{eff}}<2800 \mathrm{~K}$ ) and require new type of models. Non grey atmosphere models have been developed by Burrows et al. (1997) and Chabrier \& Baraffe (1997). Dust-free atmosphere models agree well with stellar objects down to M stars, but the dust needs to be taken into account for lower masses. A productive group (Baraffe et al. 1998, 2002; Chabrier et al. 2000) has calculated evolutionary models including dust in the atmosphere in order to provide the magnitudes of sub-stellar objects at different ages that could be compared directly to observations. The treatment of 

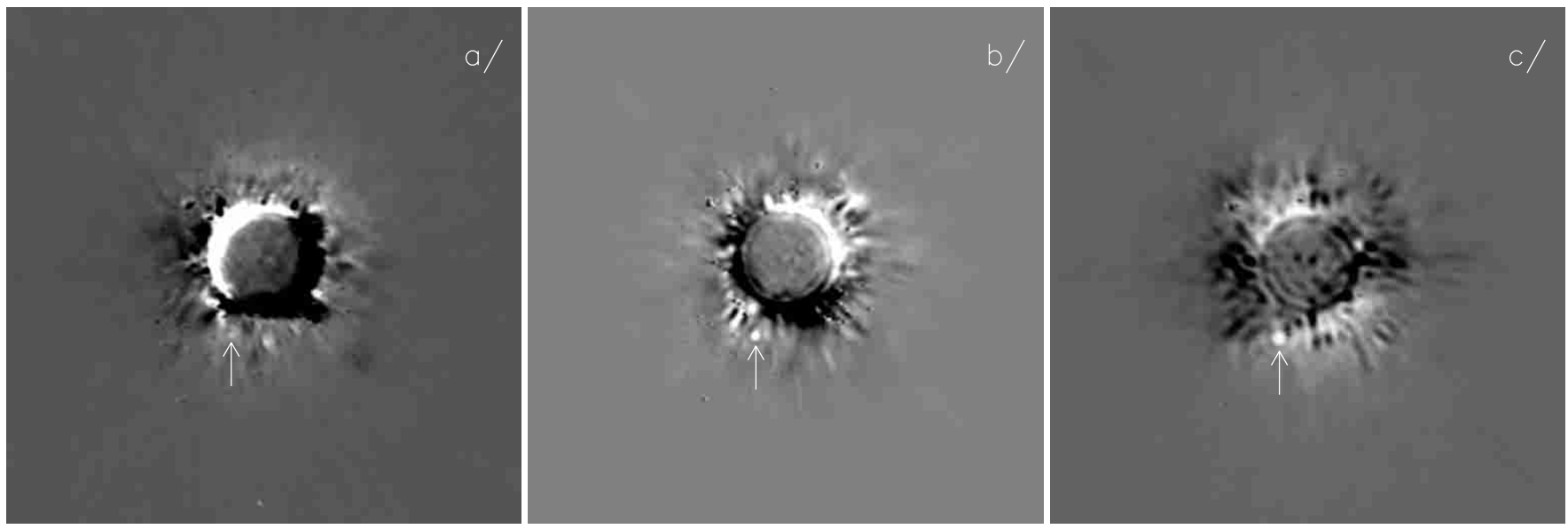

Fig. 1. Coronagraphic images of HR7672 subtracted with the calibration star HD 194012 for respectively $J\left(\right.$ a)), $H\left(\right.$ b)) and $\left.K_{\mathrm{s}}(\mathbf{c})\right)$ filters. The field of view is $5^{\prime \prime} \times 5^{\prime \prime}$. North is up, East is left.

dust is somewhat difficult and different models are not clearly distinguishable when compared to data points. In addition, the optical part of the T dwarfs spectrum is complementary to the near-IR regarding spectral typing and models were proposed by Burrows et al. (2002).

Radial velocity surveys have evidenced the so-called "Brown Dwarf desert" at small separations < 4 AU (Halbwachs et al. 2000). At very wide separations larger than $1000 \mathrm{AU}$, a few BD companions were discovered (Kirkpatrick et al. 2001) by the 2MASS Survey suggesting that this brown dwarf desert may not exist (Gizis et al. 2001). Intermediate separations can be probed by AO direct imaging as demonstrated by the case of $\mathrm{Gl} 86 \mathrm{~B}$, a possible BD orbiting a planetary system at 18.75 AU (Els et al. 2001). The problem is to know whether or not this desert is caused by a detection bias or is a direct consequence of BD formation. The gravitational influence of a $\mathrm{BD}$ close to the star may markedly impact on the history of a circumstellar disk and hence on the planetary formation (Artymowicz 1998). Increasing the sample of bounded BDs is therefore crucial for the understanding of substellar formation. Although substantial progress are still needed, high-contrast technics are efficient tools to address these basic questions.

In 2002, Liu et al. have reported the discovery of a cool low mass object as a companion candidate to the star HR 7672 using Adaptive Optics (AO) imaging. Two epochs were obtained in 2001 at Gemini-North and Keck II telescopes and the proper motion of the companion was found correlated with that of the star. Images and spectra were obtained in the $K$ band from which they derived a spectral type L4.5 \pm 1.5 . Using the theoretical models of Burrows et al. (1997) and Chabrier et al. (2000) they determined a mass of 55-78 $M_{J}$.

We obtained multi-wavelength high-contrast images of the companion to HR 7672 in order to confirm and further constrain its spectral type and mass. The results are presented in this paper. Section 2 describes the coronagraphic observations and photometric measurements are given in Sect. 3. Finally, a mass estimate is given in Sect. 4.

\section{Observations and standard reductions}

The nearby star HR 7672 (G0 V, $V=5.80$, Gray et al. 2001) was observed on July 17, 2002 at the Palomar 200 inch telescope. The data were obtained with PALAO the 241-actuators AO system (Troy et al. 2000) installed at the Cassegrain focus and PHARO the near-IR camera (Hayward. et al. 2001). A Lyot coronagraph including 2 opaque masks ( $\varnothing 0.91^{\prime \prime}$ and $\left.0.41^{\prime \prime}\right)$ with corresponding stops is located inside the cryostat of the camera and is dedicated to high-contrast imaging. The detector has a pixel sampling of 0.025 mas.

First of all, the star was observed with the $J, H$ and $K_{\mathrm{s}}$ filters without the coronagraph to provide a photometric calibration. In that case, a neutral density was necessary to avoid saturation. The Strehl ratio delivered by the AO system on that night was about $15 \%, 32 \%$ and $44 \%$ with FWHM of 51, 61 and 82 mas for respectively $J, H$ and $K_{\mathrm{s}}$ bands. Coronagraphic images of HR 7672 were then obtained with the 0.91 " mask. Both coronagraphic and non-coronagraphic images were obtained with the same pupil stop (the so-called medium cross) to allow quantitative comparison. A calibration star (HD 194012, $\mathrm{F} 8 \mathrm{~V}, V=6.17$ ) was also observed for each filter to allow subtraction of the residual static speckles. This calibration star is chosen angularly close and of similar magnitude and spectral type to ensure identical compensation by the AO system. The subtraction process is very efficient to remove the residual diffraction and the quasi-static speckles but critically depends on the precise centering of the star behind the coronagraphic mask. Images of the target and the calibration star were then corrected from the bad pixels, the flat field and were subtracted with a median sky frame. To properly perform the subtraction process, the calibration star was first recentered and then scaled to the intensity of the target. This scaling factor is derived from the coronagraphic images. The coronagraphic images of HR 7672 reduced with the above procedure are displayed in Fig. 1. The angular separation and position angle of the companion derived from the $J H K_{\mathrm{s}}$ images are $\rho=788 \pm 6$ mas and $\mathrm{PA}=156.6 \pm 0.9^{\circ}$ which is consistent with the astrometric measurements of Liu et al. (2002). 


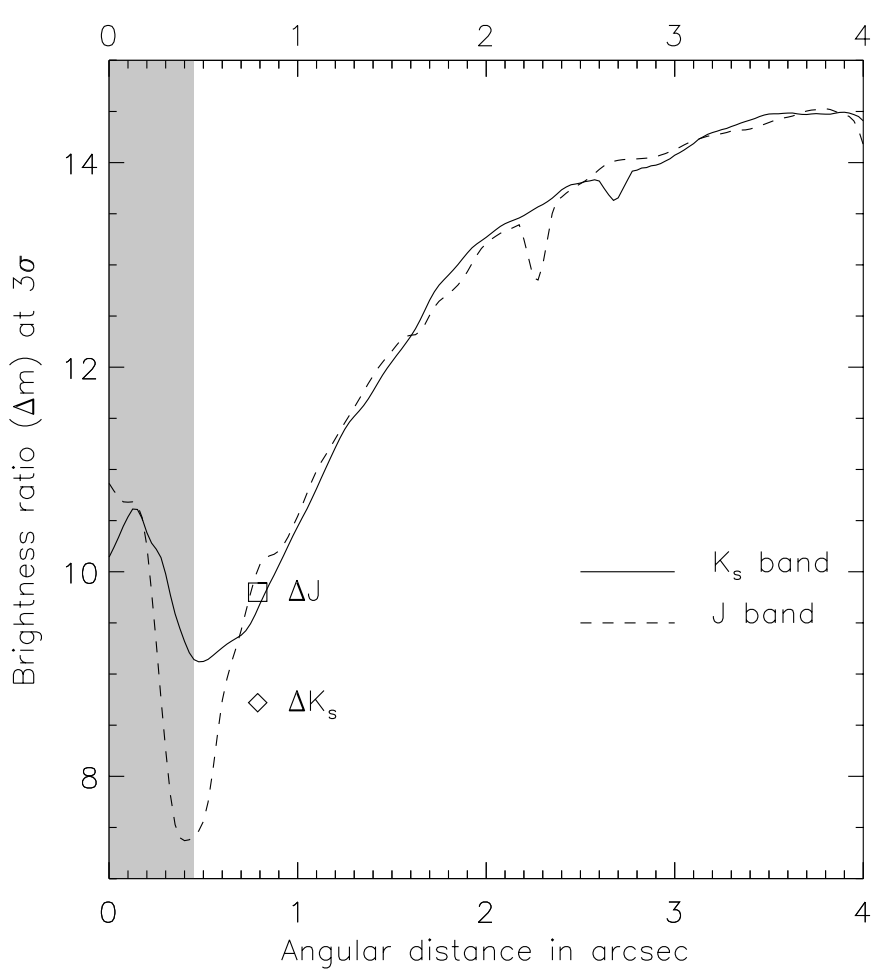

Fig. 2. Detectability of a companion in the field around the primary star. Each curve gives the contrast at $3 \sigma$ in difference of magnitudes ( $J$ and $K_{\mathrm{s}}$ ) and as a function of the angular radius. The magnitude differences $\left(\Delta J, \Delta K_{\mathrm{s}}\right)$ of the companion to HR 7672 are overplotted.

Using the coronagraphic images of Fig. 1, we compared the noise level in a ring of $\lambda / D$ at each angular radius $\rho$ with the maximum intensity of the star (no mask) to provide the companion detectability displayed of Fig. 2.

\section{Near-IR photometry of HR $7672 \mathrm{~B}$}

First of all, we measured the flux of the primary star on noncoronagraphic images. We used G1777 A, a star observed the same night, as a photometric calibrator. Its near IR magnitudes according to the Catalogue of IR Observations (Gezari et al. 1999) are $J=4.45, H=4.11$ and $K=4.05$ (with $\sim 0.02$ mag uncertainty). The fluxes of the stellar PSFs are calculated in a circular integrating window with a size ranging from $4.4 \lambda / D$ (16 pixels) to $8.8 \lambda / D$ (32 pixels) thus providing a photometric uncertainty of about $0.01 \mathrm{mag}$. Then, the corresponding photometry for HR 7672 is $J=4.59 \pm 0.04$, $H=4.40 \pm 0.02$ and $K_{\mathrm{s}}=4.32 \pm 0.02$. For the $J$ band no PSF of G1777 A was available. So, we derived the photometry of HR 7672 A using the coronagraphic data alone leading to a larger uncertainty. That probably explains the discrepancy of the color index $J-H=0.19 \pm 0.04$ with that of a standard star ( $J-H=0.305$, Bessell \& Brett 1988).

To compute the intensity of HR 7672 B we compared the flux of the primary on direct images with that of the companion detected on the coronagraphic images. The integrating window varies from $2.44 \lambda / D$ to $4.88 \lambda / D$ to derive the error bars of the HR 7672 B flux ratio. Such a small integrating window is necessary to avoid the pollution originating from
Table 1. Brightness ratio $(\Delta m)$, visual magnitude $(m)$ and absolute magnitude $(M)$ of the companion to HR 7672. (*) $J$ band photometry appears to be corrupted by the huge speckle background and is not used in this paper to derive spectral type and mass of the companion.

\begin{tabular}{cccc}
\hline \hline & $J(*)$ & $H$ & $K_{\mathrm{s}}$ \\
\hline$\Delta m$ & $9.80 \pm 0.20$ & $9.64 \pm 0.14$ & $8.72 \pm 0.10$ \\
\hline$m$ & $14.39 \pm 0.20$ & $14.04 \pm 0.14$ & $13.04 \pm 0.10$ \\
\hline$M$ & $13.16 \pm 0.20$ & $12.81 \pm 0.14$ & $11.81 \pm 0.10$ \\
\hline
\end{tabular}

coronagraphic residuals of the primary star. The measurement in the $J$ band is more critical since the peak of the companion has about the same brightness than speckle residuals from the primary and its detection was actually made possible owing to the $H$ and $K_{\mathrm{s}}$ images. In that case the integrating window was narrowed down to $1-5$ pixels in diameter. However, we are still expecting the flux measurement in the $J$ band to be overestimated. The results are summarized in Table 1 . We obtained a brightness ratio of $\Delta J=9.80 \pm 0.20, \Delta H=9.64 \pm 0.14$ and $\Delta K_{\mathrm{s}}=8.72 \pm 0.10$. The photometric uncertainty is of course much larger than for the primary star owing to the residual flux in the coronagraphic image. The corresponding color indexes are: $J-K_{\mathrm{s}}=1.35 \pm 0.22, H-K_{\mathrm{s}}=1.00 \pm 0.17$ and $J-H=0.35 \pm 0.24$.

We used the data from Dahn et al. (2002) to derive a linear relationship between the absolute $J H K_{\mathrm{s}}$ magnitudes and the spectral type of an $\mathrm{M}$ and $\mathrm{L}$ dwarfs sample. These relations are expected to be more accurate than the ones provided by Kirkpatrick et al. (2000) since they were using a smaller sample and preliminary astrometry. The fitting of this sample $(40 \mathrm{M}$ and $\mathrm{L}$ stars, $\mathrm{T}$ stars removed) leads to the following relations:

$$
\begin{aligned}
& M_{J}=8.472+0.327 \times S p \\
& M_{H}=8.212+0.279 \times S p \\
& M_{K_{\mathrm{s}}}=8.010+0.247 \times S p
\end{aligned}
$$

where $S p$ is 6.5 for M6.5 V up to 18 for L8 V. The fit dispersion is about 1.2 subclass on $X$-axis and $0.33 \mathrm{mag}$ on $Y$-axis. This gives a spectral type of L3.5 to L5 for the $J$ band (Eq. (1)), L6 to $\mathrm{L} 7$ for the $H$ band (Eq. (2)) and L5 to L6 for the $K_{\mathrm{s}}$ band (Eq. (3)). If we consider that the $J$ band photometry is corrupted by the speckle residue, the $H$ and $K_{\mathrm{s}}$ data lead to L $6 \pm 1.5$ including uncertainties and the fit scattering. Given the error bars, this result is in agreement with L4.5 \pm 1.5 announced by Liu et al. (2002).

\section{Mass estimate}

A mass estimate can be obtained from photometric measurements assuming an evolutionary model of low-mass stars. Baraffe et al. (1998) and Chabrier et al. (2000) have developed 2 types of models to describe the atmosphere of very low-mass stars. The so-called COND and DUSTY models both account for the presence of grains in the atmosphere. The DUSTY model also includes the grain opacity and is more suited for objects with $T_{\text {eff }} \lesssim 2800 \mathrm{~K}$ while the COND model 

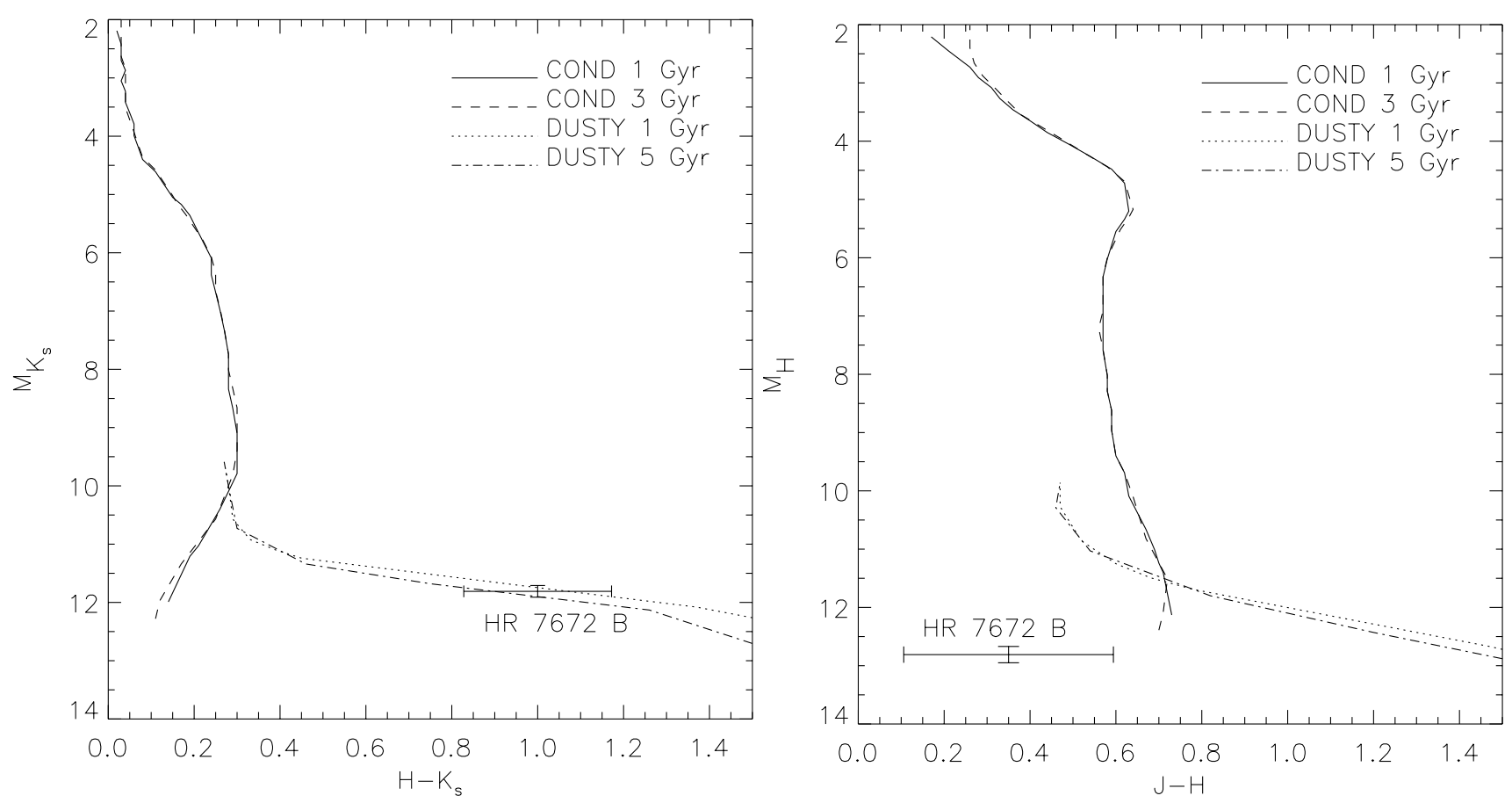

Fig. 3. Color-magnitude diagrams showing the difference between the COND and the DUSTY models of Chabrier et al. (2000). Based on the $H$ and $K_{\mathrm{s}}$ photometry we conclude that HR $7672 \mathrm{~B}$ was definitely in better agreement with the DUSTY one.

is more appropriate to describe cooler methane objects $\left(T_{\text {eff }} \lesssim\right.$ $1300-1400 \mathrm{~K})$ as explained in Baraffe et al. (2002). Then, special care is needed since HR $7672 \mathrm{~B}$ probably lies in between these temperature boundaries. Assuming an age of 1 to $3 \mathrm{Gyr}$ (Liu et al. 2002) for both the primary and the companion, we plotted the magnitude-color diagram displayed in Fig. 3. The DUSTY model appears definitely more consistent with the photometry of HR $7672 \mathrm{~B}$ at least in $H$ and $K_{\mathrm{s}}$ bands. However, the data obtained in the $J$ band are still conflicting with the models for the same reason as above.

We therefore used the isochrones of the DUSTY model (Chabrier et al. 2000) to plot the evolutionary diagrams in $J$, $H$ and $K_{\mathrm{s}}$ bands for masses ranges between $50 M_{J}$ and $80 M_{J}$ (Figs. 4, 5). The $K_{\mathrm{s}}$ and $H$ bands absolute magnitudes yield consistent values ranging between 58 and $71 M_{J}$. This slightly improves the result found by Liu et al. (2002) using $K_{\mathrm{s}}$ photometry alone $\left(55-72 M_{J}\right)$, thus revising the mass towards larger values. The $J$ band photometry is unfortunately not accurate enough and obviously leads to higher masses between 64 and $73 M_{J}$. However, although we are expecting an overestimated flux in the $J$ band, the plot of Fig. 5 shows that in this range of mass, the absolute magnitude increases very rapidly with the age. Therefore, a large photometric error does not imply systematically a large mass error. We can tentatively assess the actual $J$ band photometry assuming that HR $7672 \mathrm{~B}$ follows the same photometric relationships as field $\mathrm{L}$ dwarfs. Reversing Eq. (3), the L6 \pm 1.5 spectral type should correspond to an absolute $J$ magnitude of 13.2 to 14.2 . These new values are overplotted in Fig. 5 and give a rough estimate of the mass: 60-72 $M_{J}$ in better agreement with the mass derived from $H$ and $K_{\mathrm{s}}$.

\section{Conclusions}

Despite a lower angular resolution and a lower sensitivity than Gemini and the Keck, the high-order AO system of the Palomar 200 inch owing to the Lyot coronagraph has enable the detection of the low-mass companion to HR 7672 in the $J$, $H$ and $K_{\mathrm{s}}$ bands. This multi-wavelength photometric analysis was performed to further constrain the characteristics of the companion with respect to the previous work (Liu et al. 2002). By comparison with L field stars we derive a spectral type of L6 \pm 1.5 , although similar accuracy was obtained by Liu et al. (2002) with both $K$-band photometry and spectra. However, the 3 -colors information is required to check the consistency with the models. The color-magnitude diagrams suggest that HR $7672 \mathrm{~B}$ is better consistent with the DUSTY model of Chabrier et al. (2000) and hence, should contain dust grains in the upper atmosphere. Then, using the state-of-the-art evolutionary model we obtained a mass estimate of 58-71 $M_{J}$ based on $J, H$ and $K_{\mathrm{s}}$ photometry. This places HR $7672 \mathrm{~B}$ right below the hydrogen-burning limit. Nevertheless, as we discussed in Sect. 3, the $J$ band photometry may be questioned since the companion has an intensity very similar to that of the local speckles. A non negligible part of the stellar flux therefore contributes to the flux measurement of the companion. Additional observations, with NAOS (Lagrange et al. 2003) on the VLT for instance, would definitely provide a more accurate photometry at the shorter IR wavelengths which is mandatory to limit the possible range of mass. However, the mass derived from photometry is highly model-dependent. First, the model should be compared with a large sample of field L stars to check its consistency. Second, the case of the 2-body system HR 7672 is very interesting since it may allow to derive the dynamical 

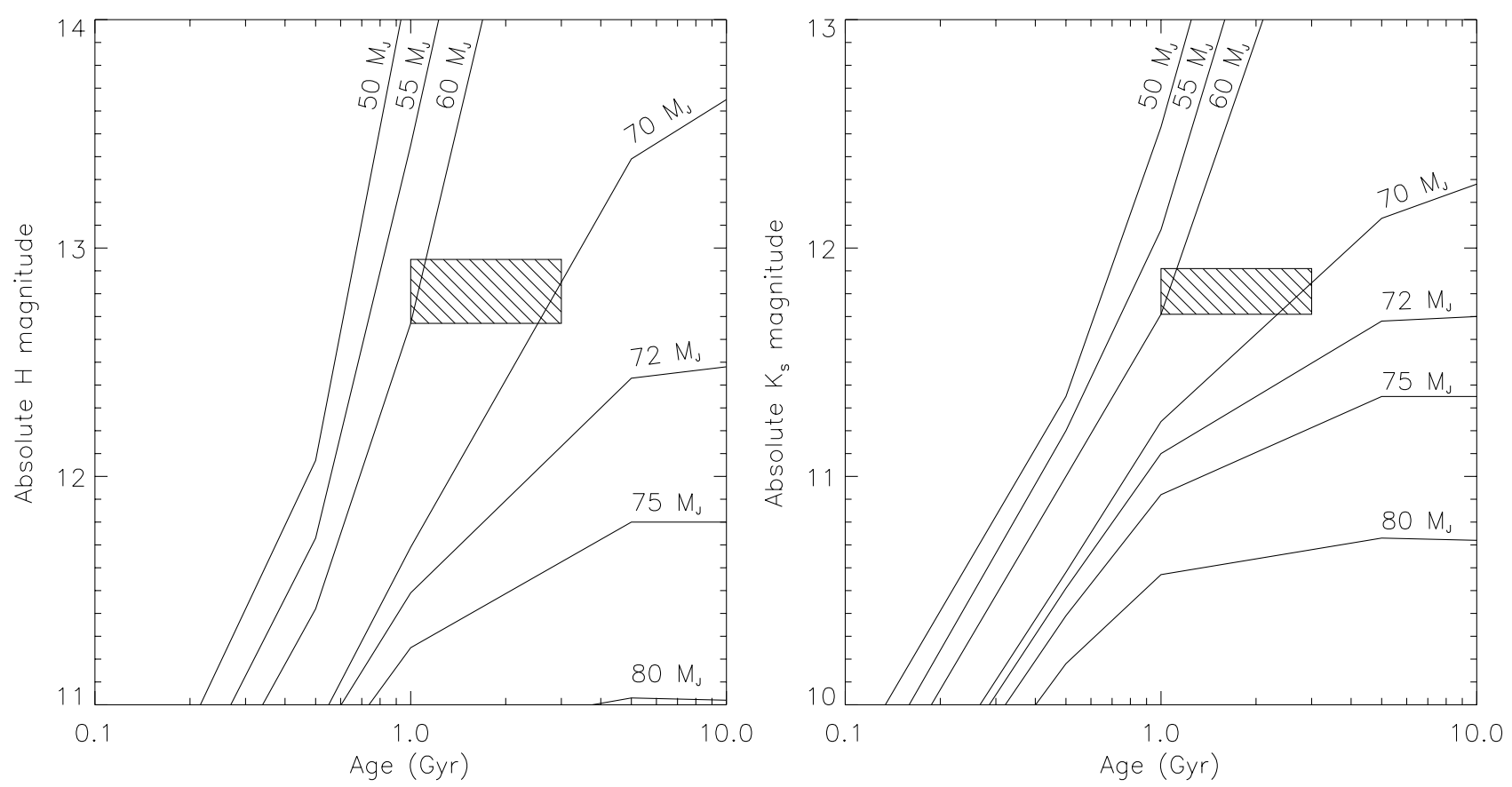

Fig. 4. Absolute magnitude in $H$ (left) and $K_{\mathrm{s}}$ (right) filters as a function of the age (in Gyr) assuming the DUSTY model of Chabrier et al. (2000). The error box for HR $7672 \mathrm{~B}$ is overplotted assuming an age of 1 to $3 \mathrm{Gyr}$.

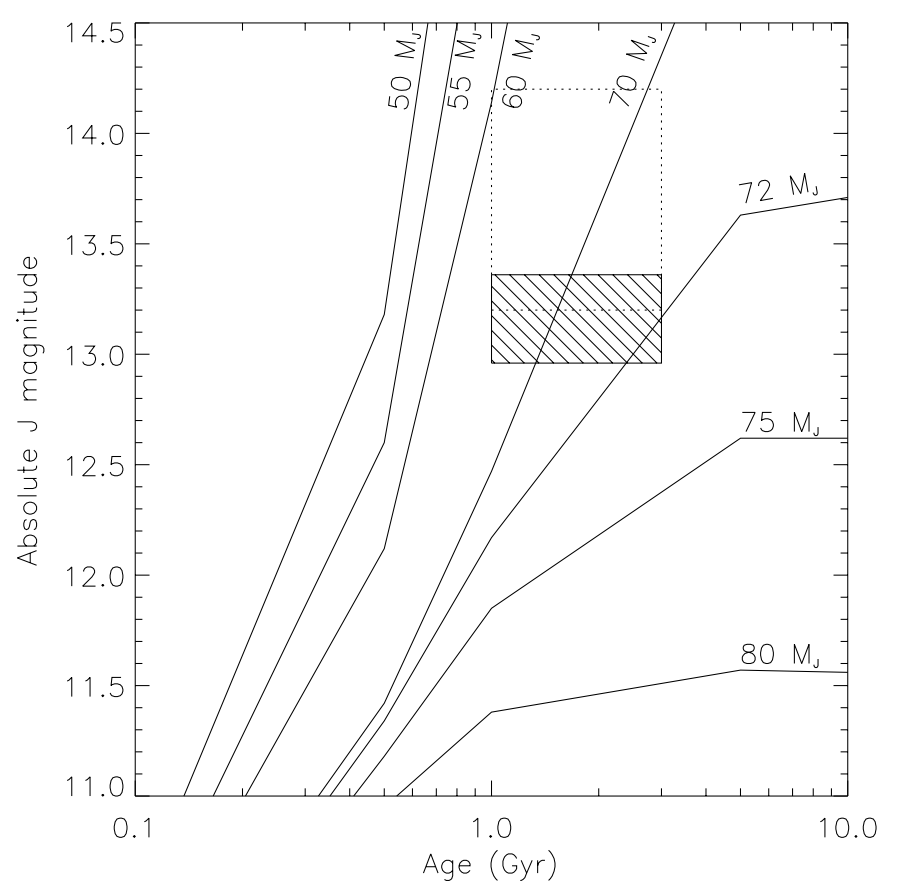

Fig. 5. Absolute magnitude in $J$ filter as a function of the age (in Gyr) assuming the DUSTY model of Chabrier et al. (2000). The error box for HR 7672 B is overplotted assuming an age of 1 to 3 Gyr. The large dash-dotted box gives the possible range of absolute $J$ magnitude consistent with the $\mathrm{L} 6 \pm 1.5$ spectral type.

mass in a few years $(r=14 \mathrm{AU})$. Coronagraphic searches of faint companions to nearby stars are therefore very important to improve the sample of binary objects.

If $\mathrm{L}$ and $\mathrm{T}$ dwarfs have been intensively observed in the near IR, very few data are available on their visible spectrum except in some particular cases (G1 229 B for instance). Dahn et al. (2002) have carried out a photometric study in the visible and near-IR of $\mathrm{L}$ and $\mathrm{T}$ dwarfs with known distances and found that the combination of visible and IR (especially $I-J$ ) is more accurate than $J H K_{\mathrm{s}}$ alone to derive the absolute magnitude of late $\mathrm{M}$ and $\mathrm{L}$ dwarfs. Therefore, visible observations of HR 7672 B would be very desirable to better understand the physics of this object. In particular, models are predicting a steep increase of the spectral energy distribution at optical wavelengths. However, L dwarfs like HR $7672 \mathrm{~B}$ are expected to be very faint in the visible $\left(m_{I}=19.59\right.$ and $\left.m_{R}=22.00\right)$ according to evolutionary models. A high-contrast imaging instrument will be required to study the L dwarf sample in the visible.

Acknowledgements. The authors would like to thank the Palomar staff for its efficient support during the observing run in July 2002, and the referee for helpful comments to improve consistency and readability of this paper.

\section{References}

Artymowicz, P. 1998, Proc. ASP Conf. Ser., 134, 152

Baraffe, I., Chabrier, G., Allard, F., \& Hauschildt, P. 1998, A\&A, 337, 403

Baraffe, I., Chabrier, G., Allard, F., \& Hauschildt, P. 2002, A\&A, 382, 563

Basri, G., Mohanty, S., Allard, F., et al. 2000, ApJ, 538, 363

Becklin, E. E., \& Zuckerman, B. 1988, Nature, 336, 656

Bessell, M., \& Brett, J. 1988, PASP, 100, 1134

Burrows, A., Burgasser, A. J., Kirkpatrick, D. J., et al. 2002, AJ, 573, 394

Burrows, A., Marley, M., Hubbard, W. B., et al. 1997, ApJ, 491, 856

Chabrier, G., \& Baraffe, I. 1997, A\&A, 327, 1039 
Chabrier, G., Baraffe, I., Allard, F., \& Hauschildt, P. 2000, ApJ, 542, 464

Dahn, C. C., Harris, H. C., Vrba, F. J., et al. 2002, AJ, 124, 1170

Els, S. G., Sterzik, M. F., Marchis, F., et al. 2001, A\&A, 370, L1

Epchtein, N. 1997, i.l.s.n. Proc., 15

Gezari, D. Y., Pitts, P. S., \& Schmitz, M. 1999, VizieR On-line Data Catalog, II/225

Gizis, J. E., Kirkpatrick, J. D., Burgasser, A., et al. 2001, ApJ, 551, 163

Gray, R., Napier, M., \& Winkler, L. 2001, AJ, 121, 2148

Halbwachs, J. L., Arenou, F., Mayor, M., Udry, S., \& Queloz, D. 2000, A\&A, 355, 581

Hayward., T. L., Brandl, B., Pirger, B., et al. 2001, PASP, 113, 105

Kirkpatrick, J. D., Dahn, C. C., Monet, D. G., et al. 2001, AJ, 121, 3235

Kirkpatrick, J. D., Reid, I. N., Liebert, J., et al. 1999, ApJ, 519, 802
Kirkpatrick, J. D., Reid, I. N., Liebert, J., et al. 2000, AJ, 120, 447

Lagrange, A., Chauvin, G., Fusco, T., et al. 2003, Proc. of the SPIE, 4841,860

Liu, M., Fischer, D., Graham, J., et al. 2002, ApJ, 571, 519

Lucas, P., \& Roche, P. 2000, MNRAS, 314, 858

Martin, E., Delfosse, X., Basri, G., et al. 1999, AJ, 118, 2466

Nakajima, T., Oppenheimer, B. R., Kulkarni, S. R., et al. 1995, Nature, 378,463

Skrutskie, M. F., Schneider, S. E., Stiening, R., et al. 19997, i.l.s.n. Proc., 25

Tinney, C., Burgasser, A., \& Kirkpatrick, J. 2003, AJ, submitted [astro-ph/0304339]

Troy, M., Dekany, R. G., Brack, G., et al. 2000, SPIE Proc., 4007, 31

York, D. G., Adelman, J., Anderson, J. E., et al. 2000, AJ, 120, 1579

Zapatero-Osorio, M. R., Béjar, V. J. S., Martín, E. L., et al. 2000, Science, 290, 103 